\title{
Strong convergence theorems and rate of convergence of multi-step iterative methods for continuous mappings on an arbitrary interval
}

\author{
Withun Phuengrattana ${ }^{1,2}$ and Suthep Suantai ${ }^{1,2^{*}}$
}

* Correspondence:
scmti005@chiangmai.ac.th
${ }^{1}$ Department of Mathematics,
Faculty of Science, Chiang Mai
University, Chiang Mai 50200,
Thailand
Full list of author information is
available at the end of the article

* Correspondence: scmti005@chiangmaiacth

Department of Mathematics, Faculty of Science, Chiang Mai

Full list of author information is

\begin{abstract}
In this article, by using the concept of $W$-mapping introduced by Atsushiba and Takahashi and K-mapping introduced by Kangtunyakarn and Suantai, we define $W^{(T}$, ${ }^{N}$-iteration and $K^{(T, M)}$-iteration for finding a fixed point of continuous mappings on an arbitrary interval. Then, a necessary and sufficient condition for the strong convergence of the proposed iterative methods for continuous mappings on an arbitrary interval is given. We also compare the rate of convergence of those iterations. It is proved that the $W^{(T, N)}$-iteration and $K^{(T, N)}$-iteration are equivalent and the $K^{(T, N)}$-iteration converges faster than the $W^{(T, N)}$-iteration. Moreover, we also present numerical examples for comparing the rate of convergence between $W^{(T, N)}$ iteration and $K^{(T, N)}$-iteration.

MSC: 26A18; 47H10; 54C05.
\end{abstract}

Keywords: fixed point, continuous mapping, $W$-mapping, $K$-mapping, rate of convergence

\section{Introduction}

There are several classical methods for approximation of solutions of nonlinear equation of one variable

$$
f(x)=0
$$

where $f: E \rightarrow E$ is a continuous function and $E$ is a closed interval on the real line. Classical fixed point iteration method is one of the methods used for this problem. To use this method, we have to transform (1.1) to the following equation:

$$
g(x)=x
$$

where $g: E \rightarrow E$ is a contraction. Then, Picard's iteration can be applied for finding a solution of (1.2).

Question: If $g: E \rightarrow E$ is continuous but not contraction, what iteration methods can be used for finding a solution of (1.2) (that is a fixed point of $g$ ) and how about the rate of convergence of those methods.

There are many iterative methods for finding a fixed point of $g$. For example, the Mann iteration (see [1]) is defined by $x_{1} \in E$ and

\section{Springer}

(C) 2012 Phuengrattana and Suantai; licensee Springer. This is an open access article distributed under the terms of the Creative Commons Attribution License (http://creativecommons.org/licenses/by/2.0), which permits unrestricted use, distribution, and reproduction in any medium, provided the original work is properly cited. 


$$
x_{n+1}=\left(1-\alpha_{n}\right) x_{n}+\alpha_{n} g\left(x_{n}\right)
$$

for all $n \geq 1$, where $\left\{\alpha_{n}\right\}_{n=1}^{\infty}$ is a sequence in [0,1]. The Ishikawa iteration (see [2]) is defined by $x_{1} \in E$ and

$$
\left\{\begin{array}{l}
y_{n}=\left(1-\beta_{n}\right) x_{n}+\beta_{n} g\left(x_{n}\right) \\
x_{n+1}=\left(1-\alpha_{n}\right) x_{n}+\alpha_{n} g\left(y_{n}\right)
\end{array}\right.
$$

for all $n \geq 1$, where $\left\{\alpha_{n}\right\}_{n=1}^{\infty},\left\{\beta_{n}\right\}_{n=1}^{\infty}$ are sequences in [0,1]. The Noor iteration (see [3]) is defined by $x_{1} \in E$ and

$$
\left\{\begin{array}{l}
z_{n}=\left(1-\gamma_{n}\right) x_{n}+\gamma_{n} g\left(x_{n}\right) \\
y_{n}=\left(1-\beta_{n}\right) x_{n}+\beta_{n} g\left(z_{n}\right) \\
x_{n+1}=\left(1-\alpha_{n}\right) x_{n}+\alpha_{n} g\left(y_{n}\right)
\end{array}\right.
$$

for all $n \geq 1$, where $\left\{\alpha_{n}\right\}_{n=1}^{\infty},\left\{\beta_{n}\right\}_{n=1}^{\infty}$, and $\left\{\gamma_{n}\right\}_{n=1}^{\infty}$ are sequences in $[0,1]$. Clearly Mann and Ishikawa iterations are special cases of Noor iteration. The SP-iteration (see [4]) is defined by $x_{1} \in E$ and

$$
\left\{\begin{array}{l}
z_{n}=\left(1-\gamma_{n}\right) x_{n}+\gamma_{n} g\left(x_{n}\right) \\
y_{n}=\left(1-\beta_{n}\right) z_{n}+\beta_{n} g\left(z_{n}\right) \\
x_{n+1}=\left(1-\alpha_{n}\right) y_{n}+\alpha_{n} g\left(y_{n}\right)
\end{array}\right.
$$

for all $n \geq 1$, where $\left\{\alpha_{n}\right\}_{n=1}^{\infty},\left\{\beta_{n}\right\}_{n=1}^{\infty}$, and $\left\{\gamma_{n}\right\}_{n=1}^{\infty}$ are sequences in [0,1]. Clearly Mann iteration is special cases of SP-iteration.

In 1976, Rhoades [5] proved the convergence of the Mann and Ishikawa iterations to a solution of (1.2) when $E=[0,1]$. He also proved the Ishikawa iteration converges faster than the Mann iteration for the class of continuous and nondecreasing functions. Later in 1991, Borwein and Borwein [6] proved the convergence of the Mann iteration of continuous functions on a bounded closed interval. In 2006, Qing and Qihou [7] extended their results to an arbitrary interval and to the Ishikawa iteration and gave some control conditions for the convergence of Ishikawa iteration on an arbitrary interval. Recently, Phuengrattana and Suantai [4] obtained a similar result for the new iteration, called the SP-iteration, and they proved the Mann, Ishikawa, Noor and SPiterations are equivalent and the SP-iteration converges faster than the others for the class of continuous and nondecreasing functions.

In this article, we are interested to employ the concept of $W$-mappings and $K$-mappings for approximation of a solution of (1.2) for a continuous function on an arbitrary interval and compare which one converges faster. The concept of $W$-mapping was first introduced by Atsushiba and Takahashi [8]. They defined $W$-mapping as follows. Let $C$ be a subset of a Banach space $X$ and $T: C \rightarrow C$ be a mapping. A point $x \in C$ is a fixed point of $T$ if $T x=x$. The set of all fixed points of $T$ is denoted by $F(T)$. Let $\left\{T_{i}\right\}_{i=1}^{N}$ be a finite family of mappings of $C$ into itself. Let $W_{n}: C \rightarrow C$ be a mapping defined by

$$
\begin{aligned}
S_{n, 0} & =I \\
S_{n, 1} & =\lambda_{n, 1} T_{1} S_{n, 0}+\left(1-\lambda_{n, 1}\right) I \\
S_{n, 2} & =\lambda_{n, 2} T_{2} S_{n, 1}+\left(1-\lambda_{n, 2}\right) I \\
& \vdots \\
S_{n, N-1} & =\lambda_{n, N-1} T_{N-1} S_{n, N-2}+\left(1-\lambda_{n, N-1}\right) I \\
W_{n}=S_{n, N} & =\lambda_{n, N} T_{N} S_{n, N-1}+\left(1-\lambda_{n, N}\right) I
\end{aligned}
$$


where $I$ is the identity mapping of $C$ and $\lambda_{n, i} \in[0,1]$ for all $i=1,2, \ldots, N$. Such a mapping $W_{n}$ is called the $W$-mapping generated by $T_{1}, T_{2}, \ldots, T_{n}$ and $\lambda_{n, 1}, \lambda_{n, 2}, \ldots, \lambda_{n, N}$. Many researchers have studied and applied this mapping for finding a common fixed point of nonexpansive mappings, for instance, see [8-23].

In 2009, Kangtunyakarn and Suantai [24] introduced a new concept of the $K$-mapping in a Banach space as follows. Let $K_{n}: C \rightarrow C$ be a mapping defined by

$$
\begin{aligned}
U_{n, 0} & =I \\
U_{n, 1} & =\lambda_{n, 1} T_{1} U_{n, 0}+\left(1-\lambda_{n, 1}\right) U_{n, 0} \\
U_{n, 2} & =\lambda_{n, 2} T_{2} U_{n, 1}+\left(1-\lambda_{n, 2}\right) U_{n, 1} \\
\vdots & \\
U_{n, N-1} & =\lambda_{n, N-1} T_{N-1} U_{n, N-2}+\left(1-\lambda_{n, N-1}\right) U_{n, N-2} \\
K_{n}=U_{n, \mathrm{~N}} & =\lambda_{n, \mathrm{~N}} T_{N} U_{n, N-1}+\left(1-\lambda_{n, \mathrm{~N}}\right) U_{n, N-1}
\end{aligned}
$$

where $I$ is the identity mapping of $C$ and $\lambda_{n, i} \in[0,1]$ for all $i=1,2, \ldots, N$. Such a mapping $K_{n}$ is called the $K$-mapping generated by $T_{1}, T_{2}, \ldots, T_{n}$ and $\lambda_{n, 1}, \lambda_{n, 2}, \ldots, \lambda_{n, N}$. They showed that if $C$ is a nonempty closed convex subset of a strictly convex Banach space $X$ and $\left\{T_{i}\right\}_{i=1}^{N}$ is a finite family of nonexpansive mappings of $C$ into itself, then $F\left(K_{n}\right)=\bigcap_{i=1}^{N} F\left(T_{i}\right)$ and they also introduced an iterative method by using the concept of $K$-mapping for finding a common fixed point of a finite family of nonexpansive mappings and a solution of an equilibrium problem. Applications of $K$-mappings for fixed point problems and equilibrium problems can be found in [23-26].

By using the concept of $W$-mappings and $K$-mappings, we introduce two new iterations for finding a fixed point of a mapping $T: E \rightarrow E$ on an arbitrary interval $E$ as follows.

The $W^{(T, N)}$-iteration is defined by $u_{1} \in E$ and

$$
u_{n+1}=W_{n}^{(T, N)} u_{n} \forall n \geq 1
$$

where $N \geq 1$ and $W_{n}^{(T, N)}$ is a mapping of $E$ into itself generated by

$$
\begin{aligned}
S_{n, 0} & =I, \\
S_{n, 1} & =\lambda_{n, 1} T S_{n, 0}+\left(1-\lambda_{n, 1}\right) I, \\
S_{n, 2} & =\lambda_{n, 2} T S_{n, 1}+\left(1-\lambda_{n, 2}\right) I, \\
\vdots & \\
S_{n, N-1} & =\lambda_{n, N-1} T S_{n, N-2}+\left(1-\lambda_{n, N-1}\right) I, \\
W_{n}^{(T, N)}=S_{n, N} & =\lambda_{n, N} T S_{n, N-1}+\left(1-\lambda_{n, N}\right) I,
\end{aligned}
$$

where $I$ is the identity mapping of $E$ and $\lambda_{n, i} \in[0,1]$ for all $i=1,2, \ldots, N$. We call a mapping $W_{n}^{(T, N)}$ as the $W$-mapping generated by $T$ and $\lambda_{n, 1}, \lambda_{n, 2}, \ldots, \lambda_{n, N}$. Clearly $W$ ${ }^{(T, 1)}$-iteration is Mann iteration, $W^{(T, 2)}$-iteration is Ishikawa iteration and $W^{(T, 3)}$-iteration is Noor iteration.

The $K^{(T, N)}$-iteration is defined by $x_{1} \in E$ and

$$
x_{n+1}=K_{n}^{(T, N)} x_{n} \forall n \geq 1,
$$


where $N \geq 1$ and $K_{n}^{(T, N)}$ is a mapping of $E$ into itself generated by

$$
\begin{aligned}
U_{n, 0} & =I \\
U_{n, 1} & =\lambda_{n, 1} T U_{n, 0}+\left(1-\lambda_{n, 1}\right) U_{n, 0}, \\
U_{n, 2} & =\lambda_{n, 2} T U_{n, 1}+\left(1-\lambda_{n, 2}\right) U_{n, 1} \\
& \vdots \\
U_{n, N-1} & =\lambda_{n, N-1} T U_{n, N-2}+\left(1-\lambda_{n, N-1}\right) U_{n, N-2,} \\
K_{n}^{(T, N)}=U_{n, N} & =\lambda_{n, N} T U_{n, N-1}+\left(1-\lambda_{n, N}\right) U_{n, N-1},
\end{aligned}
$$

where $I$ is the identity mapping of $E$ and $\lambda_{n, i} \in[0,1]$ for all $i=1,2, \ldots, N$. We call a mapping $K_{n}^{(T, N)}$ as the $K$-mapping generated by $T$ and $\lambda_{n, 1}, \lambda_{n, 2}, \ldots, \lambda_{n, N}$. Clearly $K^{(T, 1)}-$ iteration is Mann iteration and $K^{(T, 3)}$-iteration is SP-iteration.

Obviously the mappings (1.10) and (1.12) are special cases of the $W$-mapping and $K$ mapping, respectively.

The purpose of this article is to give a necessary and sufficient condition for the strong convergence of the $W^{(T, N)}$-iteration and $K^{(T, N)}$-iteration of continuous mappings on an arbitrary interval. We also prove that the $K^{(T, N)}$-iteration and $W^{(T, N)}$-iteration are equivalent and the $K^{(T, N)}$-iteration converges faster than the $W^{(T, N)}$-iteration for the class of continuous and nondecreasing mappings. Moreover, we present numerical examples for the $K^{(T, N)}$-iteration to compare with the $W^{(T, N)}$-iteration. Our results extend and improve the corresponding results of Rhoades [5], Borwein and Borwein [6], Qing and Qihou [7], Phuengrattana and Suantai [4], and many others.

\section{Convergence theorems}

We first give a convergence theorem for the $K^{(T, N)}$-iteration for continuous mappings on an arbitrary interval.

Theorem 2.1 Let $E$ be a closed interval on the real line and $T: E \rightarrow E$ be a continuous mapping. For $x_{1} \in E$, let the $K^{(T, N)}$-iteration $\left\{x_{n}\right\}_{n=1}^{\infty}$ defined by (1.11), where $\left\{\lambda_{n, i}\right\}_{n=1}^{\infty}(i=1,2, \ldots, N)$ are sequences in $[0,1]$ satisfying the following conditions:

(C1) $\sum_{n=1}^{\infty} \lambda_{n, i}<\infty$ for all $i=1,2, \ldots, N-1$;

(C2) $\lim _{n \rightarrow \infty} \lambda_{n, N}=0$ and $\sum_{n=1}^{\infty} \lambda_{n, N}=\infty$.

Then $\left\{x_{n}\right\}_{n=1}^{\infty}$ is bounded if and only if $\left\{x_{n}\right\}_{n=1}^{\infty}$ converges to a fixed point of $T$.

Proof. It is obvious that if $\left\{x_{n}\right\}_{n=1}^{\infty}$ converges to a fixed point of $T$, then it is bounded. Now, assume that $\left\{x_{n}\right\}_{n=1}^{\infty}$ is bounded. We will show that $\left\{x_{n}\right\}_{n=1}^{\infty}$ converges to a fixed point of $T$. First, we show that $\left\{x_{n}\right\}_{n=1}^{\infty}$ is convergent. To show this, we suppose not. Then there exist $a, b \in \mathbb{R}, a=\lim _{\inf _{n \rightarrow \infty}} x_{n}, b=\lim \sup _{n \rightarrow \infty} x_{n}$ and $a<b$.

Next, we show that

$$
\text { if } m \in(a, b) \text {, then } T m=m \text {. }
$$

To show this, suppose that $T m \neq m$ for some $m \in(a, b)$. Without loss of generality, we may assume that $T m-m>0$. By continuity of $T$, there exists $\delta \in(0, b-a)$ such that

$$
T x-x>0 \text { for }|x-m| \leq \delta .
$$


By boundedness of $\left\{x_{n}\right\}_{n=1}^{\infty}$, we have $\left\{x_{n}\right\}_{n=1}^{\infty}$ belongs to a bounded closed interval. Continuity of $T$ implies that $\left\{T x_{n}\right\}_{n=1}^{\infty}$ belongs to another bounded closed interval, so $\left\{T x_{n}\right\}_{n=1}^{\infty}$ is bounded. Since $U_{n, 1} x_{n}=\lambda_{n, 1} T x_{n}+\left(1-\lambda_{n, 1}\right) x_{n}$, we get $\left\{U_{n, 1} x_{n}\right\}_{n=1}^{\infty}$ is bounded, and thus $\left\{T U_{n, 1} x_{n}\right\}_{n=1}^{\infty}$ is bounded. Similarly, by using (1.11), we have $\left\{U_{n, i} x_{n}\right\}_{n=1}^{\infty}$ and $\left\{T U_{n, i} x_{n}\right\}_{n=1}^{\infty}$ are bounded for all $i=2,3, \ldots, N-1$. It follows by (1.11) that $U_{n, i} x_{n}-U_{n, i-1} x_{n}=\lambda_{n, i}\left(T U_{n, i-1} x_{n}-U_{n, i-1} x_{n}\right)$ for all $i=1,2, \ldots, N$. By condition $(C 1)$ and (C2), we get $\lim _{n \rightarrow \infty}\left|U_{n, i} x_{n}-U_{n, i-1} x_{n}\right|=0$ for all $i=1,2, \ldots, N$.

Since

$$
\begin{aligned}
\left|x_{n+1}-x_{n}\right| & =\left|U_{n, N} x_{n}-U_{n, 0} x_{n}\right| \\
& \leq\left|x_{n+1}-U_{n, N-1} x_{n}\right|+\left|U_{n, N-1} x_{n}-U_{n, N-2} x_{n}\right|+\cdots+\left|U_{n, 1} x_{n}-U_{n, 0} x_{n}\right|,
\end{aligned}
$$

it implies that $\lim _{n \rightarrow \infty}\left|x_{n+1}-x_{n}\right|=0$. Thus, there exists $M_{0}$ such that

$$
\left|x_{n+1}-x_{n}\right|<\frac{\delta}{N} \text { and }\left|U_{n, i} x_{n}-U_{n, i-1} x_{n}\right|<\frac{\delta}{N}(i=1,2, \ldots, N-1),
$$

for all $n>M_{0}$. Since $b=\lim _{\sup _{n \rightarrow \infty}} x_{n}>m$, there exists $k_{1}>M_{0}$ such that $x_{k_{1}}>m$. Let $k=k_{1}$, then $x_{k}>m$. If $x_{k} \geq m+\frac{\delta}{N}$, then by (2.3), we have $x_{k+1}>x_{k}-\frac{\delta}{N} \geq m$, so $x_{k}$ ${ }_{+1}>m$. If $x_{k} \in\left(m, m+\frac{\delta}{N}\right)$, then by (2.3), we have

$$
m-\frac{\delta}{N} i<U_{k, i} x_{k}<m+\frac{\delta}{N}(i+1) \text { for all } i=1,2, \ldots, N-1 .
$$

So we have

$$
\left|x_{k}-m\right|<\delta \text { and }\left|U_{k, i} x_{k}-m\right|<\delta \text { for all } i=1,2, \ldots, N-1 .
$$

This implies by (2.2) that

$$
T x_{k}-x_{k}>0 \text { and } T U_{k, i} x_{k}-U_{k, i} x_{k}>0 \text { for all } i=1,2, \ldots, N-1 .
$$

Using (1.11), we obtain

$$
\begin{aligned}
x_{k+1} & =\lambda_{k, N} T U_{k, N-1} x_{k}+\left(1-\lambda_{k, N}\right) U_{k, N-1} x_{k} \\
& =U_{k, N-1} x_{k}+\lambda_{k, N}\left(T U_{k, N-1} x_{k}-U_{k, N-1} x_{k}\right) \\
& =U_{k, N-2} x_{k}+\lambda_{k, N-1}\left(T U_{k, N-2} x_{k}-U_{k, N-2} x_{k}\right)+\lambda_{k, N}\left(T U_{k, N-1} x_{k}-U_{k, N-1} x_{k}\right) \\
& \vdots \\
& =x_{k}+\sum_{i=1}^{N} \lambda_{k, i}\left(T U_{k, i-1} x_{k}-U_{k, i-1} x_{k}\right) .
\end{aligned}
$$

By (2.4), we have $x_{k+1}>x_{k}$. Thus, $x_{k+1}>m$.

By using the above argument, we obtain $x_{k+j}>m$ for all $j \geq 2$. Thus we get $x_{n}>m$ for all $n>k$. So $a=\lim _{\inf _{n \rightarrow \infty}} x_{n} \geq m$, which is a contradiction with $a<m$. Thus $T m=m$. Therefore, we obtain (2.1).

For the sequence $\left\{x_{n}\right\}_{n=1}^{\infty}$, we consider the following two cases:

Case 1: There exists $x_{\bar{M}}$ such that $a<x_{\bar{M}}<b$. Then $T x_{\bar{M}}=x_{\bar{M}}$. By using (1.11), we obtain that $U_{\bar{M}, i} x_{\bar{M}}=x_{\bar{M}}$ for all $i=1,2, \ldots, N$. Thus, we have $x_{\bar{M}+1}=x_{\bar{M}}$. By induction, 
we obtain $x_{\bar{M}}=x_{\bar{M}+1}=x_{\bar{M}+2}=\ldots$, so $x_{n} \rightarrow x_{\bar{M}}$. This implies that $x_{\bar{M}}=a$ and $x_{n} \rightarrow a$, which contradicts with our assumption.

Case 2: For all $n, x_{n} \leq a$ or $x_{n} \geq b$. Because $b-a>0$ and $\lim _{n \rightarrow \infty}\left|x_{n+1}-x_{n}\right|=0$, there exists $M_{1}$ such that $\left|x_{n+1}-x_{n}\right|<\frac{b-a}{N}$ for all $n>M_{1}$. It implies that either $x_{n} \leq a$ for all $n>M_{1}$ or $x_{n} \geq b$ for all $n>M_{1}$. If $x_{n} \leq a$ for $n>M_{1}$, then $b=\lim \sup _{n \rightarrow \infty} x_{n} \leq a$, which is a contradiction with $a<b$. If $x_{n} \geq b$ for $n>M_{1}$, so we have $a=\lim \inf _{n \rightarrow \infty} x_{n}$ $\geq b$, which is a contradiction with $a<b$.

Hence, we have $\left\{x_{n}\right\}_{n=1}^{\infty}$ is convergent.

Finally, we show that $\left\{x_{n}\right\}_{n=1}^{\infty}$ converges to a fixed point of $T$. Let $\lim _{n \rightarrow \infty}, x_{n}=p$ and suppose $T p \neq p$. Since $\left\{U_{n, i} x_{n}\right\}_{n=1}^{\infty}$ is bounded for all $i=1,2, \ldots, N-1$, it implies by (1.11), condition $(C 1)$ and $(C 2)$ that $\lim _{n \rightarrow \infty} U_{n, i} x_{n}=p$ for all $i=1,2, \ldots, N-1$. Let $h_{k, i}$ $=T U_{k, i-1} x_{k}-U_{k, i-1} x_{k}$ for all $i=1,2, \ldots, N$. Continuity of $T$ implies that $\lim _{k \rightarrow \infty} h_{k, i}=T p$ - $p \neq 0$ for all $i=1,2, \ldots, N$. Put $w=T p-p$. Then $w \neq 0$. By (2.5), we have

$$
\sum_{k=1}^{n-1}\left(x_{k+1}-x_{k}\right)=\sum_{k=1}^{n-1}\left(\lambda_{k, 1} h_{k, 1}+\lambda_{k, 2} h_{k, 2}+\cdots+\lambda_{k, N} h_{k, N}\right)
$$

This implies that

$$
x_{n}=x_{1}+\sum_{k=1}^{n-1}\left(\lambda_{k, 1} h_{k, 1}+\lambda_{k, 2} h_{k, 2}+\cdots+\lambda_{k, N} h_{k, N}\right) .
$$

By condition (C1), (C2), and $\lim _{k \rightarrow \infty} h_{k, i}=w \neq 0$ for all $i=1,2, \ldots, N$, we get that $\sum_{k=1}^{\infty} \lambda_{k, i} h_{k, i}$ is convergent for all $i=1,2, \ldots, N-1$ and $\sum_{k=1}^{\infty} \lambda_{k, N} h_{k, N}$ is divergent. It follows by (2.6) that $\left\{x_{n}\right\}_{n=1}^{\infty}$ is divergent, which is a contradiction. Hence, $\left\{x_{n}\right\}_{n=1}^{\infty}$ converges to a fixed point of $T$.

We now obtain the convergence theorem of $W^{(T, N)}$-iteration. The proof is omitted because it is similar as above theorem and Theorem 2.2 of [4].

Theorem 2.2 Let $E$ be a closed interval on the real line and $T: E \rightarrow E$ be a continuous mapping. For $x_{1} \in E$, let the $W^{(T, N)}$-iteration $\left\{x_{n}\right\}_{n=1}^{\infty}$ defined by (1.9), where $\left\{\lambda_{n, i}\right\}_{n=1}^{\infty}(i=1,2, \ldots, N)$ are sequences in $[0,1]$ satisfying the following conditions:

(C1) $\lim _{n \rightarrow \infty} \lambda_{n, i}=0$ for all $i=1,2, \ldots, N$;

(C2) $\sum_{n=1}^{\infty} \lambda_{n, N}=\infty$.

Then $\left\{x_{n}\right\}_{n=1}^{\infty}$ is bounded if and only if $\left\{x_{n}\right\}_{n=1}^{\infty}$ converges to a fixed point of $T$.

The following results are obtained direclty from Theorem 2.1.

Corollary 2.3 ([4, Theorem 2.1]) Let E be a closed interval on the real line and $T$ : $E \rightarrow E$ be a continuous mapping. For $x_{1} \in E$, let the SP-iteration $\left\{x_{n}\right\}_{n=1}^{\infty}$ defined by (1.6), where $\left\{\lambda_{n, 1}\right\}_{n=1}^{\infty},\left\{\lambda_{n, 2}\right\}_{n=1}^{\infty}$, and $\left\{\lambda_{n, 3}\right\}_{n=1}^{\infty}$ are sequences in $[0,1]$ satisfying the following conditions:

(C1) $\sum_{n=1}^{\infty} \lambda_{n, 1}<\infty$ and $\sum_{n=1}^{\infty} \lambda_{n, 2}<\infty$;

(C2) $\lim _{n \rightarrow \infty} \lambda_{n, 3}=0$ and $\sum_{n=1}^{\infty} \lambda_{n, 3}=\infty$.

Then $\left\{x_{n}\right\}_{n=1}^{\infty}$ is bounded if and only if $\left\{x_{n}\right\}_{n=1}^{\infty}$ converges to a fixed point of $T$.

Corollary 2.4 ([7, Theorem 3]) Let E be a closed interval on the real line and $T: E$ $\rightarrow E$ be a continuous mapping. For $x_{1} \in E$, let the Mann iteration $\left\{x_{n}\right\}_{n=1}^{\infty}$ defined by 
(1.3), where $\left\{\lambda_{n, 1}\right\}_{n=1}^{\infty}$ is a sequence in $[0,1]$ satisfying $\lim _{n \rightarrow \infty}, \lambda_{n, 1}=0$ and $\sum_{n=1}^{\infty} \lambda_{n, 1}=\infty$. Then $\left\{x_{n}\right\}_{n=1}^{\infty}$ is bounded if and only if $\left\{x_{n}\right\}_{n=1}^{\infty}$ converges to a fixed point of $T$.

The following results are obtained directly from Theorem 2.2.

Corollary 2.5 ([4, Theorem 2.2]) Let E be a closed interval on the real line and $T$ : $E \rightarrow E$ be a continuous mapping. For $x_{1} \in E$, let the Noor iteration $\left\{x_{n}\right\}_{n=1}^{\infty}$ defined by (1.5), where $\left\{\lambda_{n, 1}\right\}_{n=1}^{\infty},\left\{\lambda_{n, 2}\right\}_{n=1}^{\infty},\left\{\lambda_{n, 3}\right\}_{n=1}^{\infty}$ are sequences in $[0,1]$ satisfying the following conditions:

(C1) $\lim _{n \rightarrow \infty} \lambda_{n, 1}=0, \lim _{n \rightarrow \infty} \lambda_{n, 2}=0$ and $\lim _{n \rightarrow \infty} \lambda_{n, 3}=0$;

(C2) $\sum_{n=1}^{\infty} \lambda_{n, 3}=\infty$.

Then $\left\{x_{n}\right\}_{n=1}^{\infty}$ is bounded if and only if $\left\{x_{n}\right\}_{n=1}^{\infty}$ converges to a fixed point of $T$.

Corollary 2.6 ([7]) Let $E$ be a closed interval on the real line and $T: E \rightarrow E$ be a continuous mapping. For $x_{1} \in E$, let the Ishikawa iteration $\left\{x_{n}\right\}_{n=1}^{\infty}$ defined by (1.4), where $\left\{\lambda_{n, 1}\right\}_{n=1}^{\infty}$ are sequences in $[0,1]$ satisfying the following conditions:

(C1) $\lim _{n \rightarrow \infty} \lambda_{n, 1}=0$ and $\lim _{n \rightarrow \infty} \lambda_{n, 2}=0$;

(C2) $\sum_{n=1}^{\infty} \lambda_{n, 2}=\infty$.

Then $\left\{x_{n}\right\}_{n=1}^{\infty}$ is bounded if and only if $\left\{x_{n}\right\}_{n=1}^{\infty}$ converges to a fixed point of $T$.

\section{Rate of convergence and numerical examples}

There are many articles have been published on the iterative methods using for approximation of fixed points of nonlinear mappings, see for instance [1-7]. However, there are only a few articles concerning comparison of those iterative methods in order to establish which one converges faster. As far as we know, there are two ways for comparison of the rate of convergence. The first one was introduced by Berinde [27]. He used this idea to compare the rate of convergence of Picard and Mann iterations for a class of Zamfirescu operators in arbitrary Banach spaces. Popescu [28] also used this concept to compare the rate of convergence of Picard and Mann iterations for a class of quasi-contractive operators. It was shown in [29] that the Mann and Ishikawa iterations are equivalent for the class of Zamfirescu operators. In 2006, Babu and Prasad [30] showed that the Mann iteration converges faster than the Ishikawa iteration for this class of operators. Two years later, Qing and Rhoades [31] provided an example to show that the claim of Babu and Prasad [30] is false.

However, this concept is not suitable or cannot be applied to a class of continuous self-mappings defined on a closed interval. In order to compare the rate of convergence of continuous self-mappings defined on a closed interval, Rhoades [5] introduced the other concept which is slightly different from that of Berinde to compare iterative methods which one converges faster as follows.

Definition 3.1 Let $E$ be a closed interval on the real line and $T: E \rightarrow E$ be a continuous mapping. Suppose that $\left\{x_{n}\right\}_{n=1}^{\infty}$ and $\left\{u_{n}\right\}_{n=1}^{\infty}$ are two iterations which converge to the fixed point $p$ of $T$. We say that $\left\{x_{n}\right\}_{n=1}^{\infty}$ converges faster than $\left\{u_{n}\right\}_{n=1}^{\infty}$ if

$$
\left|x_{n}-p\right| \leq\left|u_{n}-p\right| \text { for all } n \geq 1 \text {. }
$$


In this section, we study the rate of convergence of $W^{(T, N)}$-iteration and $K^{(T, N)}$-iteration for continuous and nondecreasing mappings on an arbitrary interval in the sense of Rhoades. The following lemmas are useful and crucial for our following results.

Lemma 3.2 Let $E$ be a closed interval on the real line and $T: E \rightarrow E$ be a continuous and nondecreasing mapping such that $F(T)$ is nonempty and bounded with $x_{1}>\sup \{p$ $\in E: p=T p\}$. Let $\left\{x_{n}\right\}_{n=1}^{\infty}$ be defined by $W^{(T, N)}$-iteration or $K^{(T, N)}$-iteration. If $T x_{1}>x_{1}$, then $\left\{x_{n}\right\}_{n=1}^{\infty}$ does not converge to a fixed point of $T$.

Proof. We prove only the case that $\left\{x_{n}\right\}_{n=1}^{\infty}$ is defined by $K^{(T, N)}$-iteration because the other case can be proved similarly.

Let $T x_{1}>x_{1}$. Since $x_{1}>\sup \{p \in E: p=T p\}$ and by using (1.11) and mathematical induction, we can show that $x_{n} \geq \sup \{p \in E: p=T p\}$ for all $n \geq 1$. It is clear that $T x_{n}$ $\geq x_{n}$ for all $n \geq 1$. Using (1.11), we have

$$
U_{n, 1} x_{n}=\lambda_{n, 1} T x_{n}+\left(1-\lambda_{n, 1}\right) x_{n} \geq x_{n} \text { for all } n \geq 1 .
$$

Since $T$ is nondecreasing, we have $T U_{n, 1} x_{n} \geq T x_{n} \geq x_{n}$. Using (1.11) again, we have

$$
U_{n, 2} x_{n}=\lambda_{n, 2} T U_{n, 1} x_{n}+\left(1-\lambda_{n, 2}\right) U_{n, 1} x_{n} \geq x_{n} \text { for all } n \geq 1 .
$$

This implies that $T U_{n, 2} x_{n} \geq T x_{n} \geq x_{n}$. By continuity in this way, we can show that $x_{n+1}=K_{n}^{(T, N)} x_{n}=U_{n, N} x_{n} \geq x_{n}$ for all $n \geq 1$. Thus $\left\{x_{n}\right\}_{n=1}^{\infty}$ is nondecreasing. But $x_{1}>$ $\sup \{p \in E: p=T p\}$, it implies that $\left\{x_{n}\right\}_{n=1}^{\infty}$ does not converges to a fixed point of $T$.

By using the same argument of proof as in above lemma, we get the following result.

Lemma 3.3 Let $E$ be a closed interval on the real line and $T: E \rightarrow E$ be a continuous and nondecreasing mapping such that $F(T)$ is nonempty and bounded with $x_{1}<\inf \{p \in$ $E: p=T p\}$. Let $\left\{x_{n}\right\}_{n=1}^{\infty}$ be defined by $W^{(T, N)}$-iteration or $K^{(T, N)}$-iteration. If $T x_{1}<x_{1}$, then $\left\{x_{n}\right\}_{n=1}^{\infty}$ does not converge to a fixed point of $T$.

We now get the following theorem for compare rate of convergence between $W^{(T, N)}$ iteration and $K^{(T, N)}$-iteration.

Theorem 3.4 Let $E$ be a closed interval on the real line and $T: E \rightarrow E$ be a continuous and nondecreasing mapping such that $F(T)$ is nonempty and bounded. For $u_{1}=x_{1}$ $\in E$, let $\left\{u_{n}\right\}_{n=1}^{\infty}$ and $\left\{x_{n}\right\}_{n=1}^{\infty}$ are the sequences defined by (1.9) and (1.11), respectively. Let $\left\{\lambda_{n, i}\right\}_{n=1}^{\infty}$ be sequences in $[0,1)$ for all $i=1,2, \ldots, N$. Then, the $W^{(T, N)}$-iteration $\left\{u_{n}\right\}_{n=1}^{\infty}$ converges to the fixed point $p$ of $T$ if and only if the $K^{(T, N)}$-iteration $\left\{x_{n}\right\}_{n=1}^{\infty}$ converges to p. Moreover, the $K^{(T, N)}$-iteration converges faster than the $W^{(T, N)}$-iteration.

Proof. Put $L=\inf \{p \in E: p=T p\}$ and $U=\sup \{p \in E: p=T p\}$.

$\Leftrightarrow$ Suppose that the $W^{(T, N)}$-iteration $\left\{u_{n}\right\}_{n=1}^{\infty}$ converges to the fixed point $p$ of $T$.

We divide our proof into the following three cases:

Case 1: $u_{1}=x_{1}>U$. By Lemma 3.2, we have $T u_{1}<u_{1}$ and $T x_{1}<x_{1}$. We now show that $x_{n} \leq u_{n}$ for all $n \geq 1$. Assume that $x_{k} \leq u_{k}$. Thus, $T x_{k} \leq T u_{k}$. Since $x_{1}>U$ and by using (1.11) and mathematical induction, we can show that $x_{n} \geq U$ for all $n \geq 1$. It is clear that $T x_{k} \leq x_{k}$. This implies that $T x_{k} \leq U_{k, 1} x_{k} \leq x_{k}$. Since $T$ is nondecreasing, $T U_{k, 1} x_{k} \leq$ $T x_{k}$. Thus, we have

$$
T U_{k, 1} x_{k} \leq U_{k, 2} x_{k} \leq U_{k, 1} x_{k} .
$$


It follows that $U_{k, 2} x_{k} \leq x_{k}$. By (3.1) and $T$ is nondecreasing, we have $T U_{k, 2} x_{k} \leq T U_{k, 1}$ $x_{k} \leq U_{k, 2} x_{k}$. This implies that

$$
T U_{k, 2} x_{k} \leq U_{k, 3} x_{k} \leq U_{k, 2} x_{k}
$$

Thus, we have $U_{k, 3} x_{k} \leq x_{k}$. By continuity in this way, we can show that

$$
U_{k, i} x_{k} \leq x_{k} \text { for all } i=1,2, \ldots, N \text {. }
$$

Using (1.9) and (1.11), we get

$$
U_{k, 1} x_{k}-S_{k, 1} u_{k}=\lambda_{k, 1}\left(x_{k}-u_{k}\right)+\left(1-\lambda_{k, 1}\right)\left(T x_{k}-T u_{k}\right) \leq 0 .
$$

Since $T$ is nondecreasing, we have $T U_{k, 1} x_{k} \leq T S_{k, 1} u_{k}$. It follows that

$$
\begin{aligned}
U_{k, 2} x_{k}-S_{k, 2} u_{k} & =\lambda_{k, 2}\left(U_{k, 1} x_{k}-u_{k}\right)+\left(1-\lambda_{k, 2}\right)\left(T U_{k, 1} x_{k}-T S_{k, 1} u_{k}\right) \\
& \leq \lambda_{k, 2}\left(U_{k, 1} x_{k}-x_{k}\right)+\left(1-\lambda_{k, 2}\right)\left(T U_{k, 1} x_{k}-T S_{k, 1} u_{k}\right) \\
& \leq 0 .
\end{aligned}
$$

That is $U_{k, 2} x_{k} \leq S_{k, 2} u_{k}$. Since $T$ is nondecreasing, we have $T U_{k, 2} x_{k} \leq T S_{k, 2} u_{k}$. This implies that

$$
\begin{aligned}
U_{k, 3} x_{k}-S_{k, 3} u_{k} & =\lambda_{k, 3}\left(U_{k, 2} x_{k}-u_{k}\right)+\left(1-\lambda_{k, 3}\right)\left(T U_{k, 2} x_{k}-T S_{k, 2} u_{k}\right) \\
& \leq \lambda_{k, 3}\left(U_{k, 2} x_{k}-x_{k}\right)+\left(1-\lambda_{k, 3}\right)\left(T U_{k, 2} x_{k}-T S_{k, 2} u_{k}\right) \\
& \leq 0 .
\end{aligned}
$$

That is $U_{k, 3} x_{k} \leq S_{k, 3} u_{k}$. By continuity in this way we can show that $U_{k, N} x_{k} \leq S_{k, N} u_{k}$. Thus, $x_{k+1} \leq u_{k+1}$. Hence, by mathematical induction, we obtain $x_{n} \leq u_{n}$ for all $n \geq 1$. By $x_{n} \geq U$ for all $n \geq 1$, we get $0 \leq x_{n}-p \leq u_{n}-p$, so

$$
\left|x_{n}-p\right| \leq\left|u_{n}-p\right| \text { for all } n \geq 1 \text {. }
$$

Since $\lim _{n \rightarrow \infty}, u_{n}=p$, it implies that $\lim _{n \rightarrow \infty} x_{n}=p$. That is, the $K^{(T, N)}$-iteration $\left\{x_{n}\right\}_{n=1}^{\infty}$ converges to the same fixed point $p$. Moreover, by (3.2), we see that the $K^{(T, N)}$ iteration $\left\{x_{n}\right\}_{n=1}^{\infty}$ converges faster than the $W^{(T, N)}$-iteration $\left\{u_{n}\right\}_{n=1}^{\infty}$.

Case 2: $u_{1}=x_{1}<L$. By Lemma 3.3, we have $T u_{1}>u_{1}$ and $T x_{1}>x_{1}$. By using (1.9), (1.11) and the same argument as in Case 1 , we can show that $x_{n} \geq u_{n}$ for all $n \geq 1$. We note that $x_{1}<L$ and by using (1.11) and mathematical induction, we can show that $x_{n} \leq$ $L$ for all $n \geq 1$. Thus, we have $\left|x_{n}-p\right| \leq\left|u_{n}-p\right|$ for all $n \geq 1$. It follows that $\lim _{n \rightarrow \infty}$ $x_{n}=p$ and the $K^{(T, N)}$-iteration $\left\{x_{n}\right\}_{n=1}^{\infty}$ converges faster than the $W^{(T, N)}$-iteration $\left\{u_{n}\right\}_{n=1}^{\infty}$.

Case 3: $L \leq u_{1}=x_{1} \leq U$. Suppose that $T u_{1} \neq u_{1}$. Without loss of generality, we suppose $T u_{1}<u_{1}$. It follows by (1.9) that $u_{n} \leq u_{1}$ for all $n \geq 1$. Since $\lim _{n \rightarrow \infty} u_{n}=p$, we must get $p<u_{1}=x_{1}$. By the same argument as in Case 1 , we have $p \leq x_{n} \leq u_{n}$ for all $n$ $\geq 1$. It follows that $\left|x_{n}-p\right| \leq\left|u_{n}-p\right|$ for all $n \geq 1$. Hence, $\lim _{n \rightarrow \infty} x_{n}=p$ and the $K^{(T,}$ ${ }^{N)}$-iteration $\left\{x_{n}\right\}_{n=1}^{\infty}$ converges faster than the $W^{(T, N)}$-iteration $\left\{u_{n}\right\}_{n=1}^{\infty}$.

$(\Leftarrow)$ Suppose that the $K^{(T, N)}$-iteration $\left\{x_{n}\right\}_{n=1}^{\infty}$ converges to the fixed point $p$ of $T$. Put $\lambda_{n, i}=0$ for all $i=1,2, \ldots, N-1$ and $n \geq 1$, we get the sequence $\left\{x_{n}\right\}_{n=1}^{\infty}$ generated by

$$
x_{n+1}=\lambda_{n, N} T x_{n}+\left(1-\lambda_{n, N}\right) x_{n} \text { for all } n \geq 1
$$


that converges to $p$. We will show that $W^{(T, N)}$-iteration $\left\{u_{n}\right\}_{n=1}^{\infty}$ converges to $p$. We shall prove only the case $x_{1}=u_{1}>U$, because other cases can be proved similarly as the first part. By Proposition 3.5 in [4], we get $T x_{1}<x_{1}$ and $T u_{1}<u_{1}$. Assume that $u_{k}$ $\leq x_{k}$. Thus $T u_{k} \leq T x_{k}$. Since $u_{1}>U$ and by using (1.9) and mathematical induction, we can show that $u_{n} \geq U$ for all $n \geq 1$. It is clear that $T u_{k} \leq u_{k}$. This implies that $T u_{k} \leq$ $S_{k, 1} u_{k} \leq u_{k}$. Since $T$ is nondecreasing, $T S_{k, 1} u_{k} \leq T u_{k} \leq S_{k, 1} u_{k}$. Thus, $T S_{k, 1} u_{k} \leq u_{k} \leq x_{k}$. It follows that $T S_{k, 1} u_{k} \leq S_{k, 2} u_{k} \leq u_{k}$. Since $T$ is nondecreasing, $T S_{k, 2} u_{k} \leq T u_{k} \leq S_{k, 1} u_{k}$. Thus, $T S_{k, 2} u_{k} \leq u_{k} \leq x_{k}$. By continuity in this way, we have $T S_{k, i} u_{k} \leq x_{k}$ for all $i=1$, $2, \ldots, N$. By (1.9) and (3.3), we obtain

$$
S_{k, i} u_{k}-x_{k}=\lambda_{k, i}\left(u_{k}-x_{k}\right)+\left(1-\lambda_{k, i}\right)\left(T S_{k, i-1} u_{k}-x_{k}\right) \leq 0,
$$

for all $i=2,3, \ldots, N-1$. Since $T$ is nondecreasing, we have

$$
T S_{k, i} u_{k} \leq T x_{k} \text { for all } i=2,3, \ldots, N-1 \text {. }
$$

It follows by (1.9) and (3.3) that

$$
u_{k+1}-x_{k+1}=\lambda_{k, N}\left(u_{k}-x_{k}\right)+\left(1-\lambda_{k, N}\right)\left(T S_{k, N-1} u_{k}-T x_{k}\right) \leq 0 .
$$

By mathematical induction, we have $u_{n} \leq x_{n}$ for all $n \geq 1$. We note that $x_{1}>U$ and by using (3.3) and mathematical induction, we can show that $x_{n} \geq U$ for all $n \geq 1$. Thus, we have $0 \leq u_{n}-p \leq x_{n}-p$ for all $n \geq 1$. Since $\lim _{n \rightarrow \infty} x_{n}=p$, it follows that $\lim _{n \rightarrow \infty}$ $u_{n}=p$ That is, the $W^{(T, N)}$-iteration $\left\{u_{n}\right\}_{n=1}^{\infty}$ converges to the same fixed point $p$.

We also consider the speed of convergence of the $K^{(T, N)}$-iteration which depends on the choice of control sequences $\left\{\lambda_{n, i}\right\}_{n=1}^{\infty}(i=1,2, \ldots, N)$ as the following theorem.

Theorem 3.5 Let $E$ be a closed interval on the real line and $T: E \rightarrow E$ be a continuous and nondecreasing mapping such that $F(T)$ is nonempty and bounded. Let $\left\{\lambda_{n, i}\right\}_{n=1}^{\infty}$, $\left\{\lambda_{n, i}^{*}\right\}_{n=1}^{\infty}$ are the sequences in $[0,1)$ such that $\lambda_{n, i} \leq \lambda_{n, i}^{*}$ for all $i=1,2, \ldots$, . N. Let $\left\{x_{n}\right\}_{n=1}^{\infty}$ be a sequence defined by $x_{1} \in E$ and

$$
x_{n+1}=K_{n}^{(T, N)} x_{n} \forall n \geq 1,
$$

where $K_{n}^{(T, N)}$ is the K-mapping generated by $T$ and $\lambda_{n, 1}, \lambda_{n, 2}, \ldots, \lambda_{n, N}$, and $\left\{x_{n}^{*}\right\}_{n=1}^{\infty}$ be a sequence defined by $x_{1}^{*}=x_{1} \in E$ and

$$
x_{n+1}^{*}=\bar{K}_{n}^{(T, N)} x_{n}^{*} \forall n \geq 1,
$$

where $\bar{K}_{n}^{(T, N)}$ is the K-mapping generated by $T$ and $\lambda_{n, 1}^{*}, \lambda_{n, 2}^{*}, \ldots, \lambda_{n, N}^{*}$.

If $\left\{x_{n}\right\}_{n=1}^{\infty}$ converges to the fixed point $p$ of $T$, then $\left\{x_{n}^{*}\right\}_{n=1}^{\infty}$ converges to $p$. Moreover, $\left\{x_{n}^{*}\right\}_{n=1}^{\infty}$ converges faster than $\left\{x_{n}\right\}_{n=1}^{\infty}$.

Proof. Put $L=\inf \{p \in E: p=T p\}$ and $U=\sup \{p \in E: p=T p\}$. Suppose that $\left\{x_{n}\right\}_{n=1}^{\infty}$ converges to a fixed point $p$ of $T$. We divide our proof into the following three cases:

Case 1: $x_{1}^{*}=x_{1}>U$. By Lemma 3.2, we have $T x_{1}^{*}<x_{1}^{*}$ and $T x_{1}<x_{1}$. Assume that $T x_{k}^{*} \leq U_{k, 1} x_{k}^{*} \leq x_{k}^{*}$. Thus, $T x_{k}^{*} \leq T x_{k}^{*}$. Since $x_{1}^{*}>U$ and by using (3.5) and 
mathematical induction, we can show that $x_{n}^{*} \geq U$ for all $n \geq 1$. It is clear that $T x_{k}^{*} \leq x_{k}^{*}$. This implies that $T x_{k}^{*} \leq U_{k, 1} x_{k}^{*} \leq x_{k}^{*}$. Since $T$ is nondecreasing, $T U_{k, 1} x_{k}^{*} \leq T x_{k}^{*}$. Thus, we have

$$
T U_{k, 1} x_{k}^{*} \leq U_{k, 2} x_{k}^{*} \leq U_{k, 1} x_{k}^{*} .
$$

It follows that $T U_{k, 2} x_{k}^{*} \leq T U_{k, 1} x_{k}^{*} \leq U_{k, 2} x_{k}^{*}$. This implies that

$$
T U_{k, 2} x_{k}^{*} \leq U_{k, 3} x_{k}^{*} \leq U_{k, 2} x_{k}^{*} .
$$

By continuity in this way, we can show that

$$
T U_{k, i} x_{k}^{*} \leq U_{k, i} x_{k}^{*} \text { for all } i=0,1, \ldots, N .
$$

Using (3.4), (3.5), and (3.6), we have

$$
\begin{aligned}
U_{k, 1} x_{k}^{*}-U_{k, 1} x_{k} & =\left(U_{k, 0} x_{k}^{*}-U_{k, 0} x_{k}\right)+\lambda_{k, 1}^{*}\left(T U_{k, 0} x_{k}^{*}-U_{k, 0} x_{k}^{*}\right)+\lambda_{k, 1}\left(U_{k, 0} x_{k}-T U_{k, 0} x_{k}\right) \\
& \leq\left(U_{k, 0} x_{k}^{*}-U_{k, 0} x_{k}\right)+\lambda_{k, 1}^{*}\left(T U_{k, 0} x_{k}^{*}-U_{k, 0} x_{k}^{*}\right)+\lambda_{k, 1}^{*}\left(U_{k, 0} x_{k}-T U_{k, 0} x_{k}\right) \\
& =\left(1-\lambda_{k, 1}^{*}\right)\left(U_{k, 0} x_{k}^{*}-U_{k, 0} x_{k}\right)+\lambda_{k, 1}^{*}\left(T U_{k, 0} x_{k}^{*}-T U_{k, 0} x_{k}\right) \\
& \leq 0 .
\end{aligned}
$$

This implies $T U_{k, 1} x_{k}^{*} \leq T U_{k, 1} x_{k}$. It follows that

$$
\begin{aligned}
U_{k, 2} x_{k}^{*}-U_{k, 2} x_{k} & =\left(U_{k, 1} x_{k}^{*}-U_{k, 1} x_{k}\right)+\lambda_{k, 2}^{*}\left(T U_{k, 1} x_{k}^{*}-U_{k, 1} x_{k}^{*}\right)+\lambda_{k, 2}\left(U_{k, 1} x_{k}-T U_{k, 1} x_{k}\right) \\
& \leq\left(U_{k, 1} x_{k}^{*}-U_{k, 1} x_{k}\right)+\lambda_{k, 2}^{*}\left(T U_{k, 1} x_{k}^{*}-U_{k, 1} x_{k}^{*}\right)+\lambda_{k, 2}^{*}\left(U_{k, 1} x_{k}-T U_{k, 1} x_{k}\right) \\
& =\left(1-\lambda_{k, 2}^{*}\right)\left(U_{k, 1} x_{k}^{*}-U_{k, 1} x_{k}\right)+\lambda_{k, 2}^{*}\left(T U_{k, 1} x_{k}^{*}-T U_{k, 1} x_{k}\right) \\
& \leq 0 .
\end{aligned}
$$

By continuity in this way, we can show that

$$
\bar{K}_{k}^{(T, N)} x_{k}^{*}-K_{k}^{(T, N)} x_{k}=U_{k, N} x_{k}^{*}-U_{k, N} x_{k} \leq 0 .
$$

That is, $x_{k+1}^{*} \leq x_{k+1}$. By mathematical induction, we obtain $x_{n}^{*} \leq x_{n}$ for all $n \geq 1$. Since $x_{n}^{*} \geq U$ for all $n \geq 1$, we get $0 \leq x_{n}^{*}-p \leq x_{n}-p$, so $\left|x_{n}^{*}-p\right| \leq\left|x_{n}-p\right|$ for all $n$ $\geq 1$. It follows that $\lim _{n \rightarrow \infty} x_{n}^{*}=p$ and $\left\{x_{n}^{*}\right\}_{n=1}^{\infty}$ converges faster than $\left\{x_{n}\right\}_{n=1}^{\infty}$.

Case 2: $x_{1}^{*}=x_{1}<L$. By Lemma 3.3, we have $T x_{1}^{*}>x_{1}^{*}$ and $T x_{1}>x_{1}$. By using (3.4), (3.5) and the same argument as in Case 1 , we can show that $x_{n}^{*} \geq x_{n}$ for all $n \geq 1$. We note that $x_{1}^{*}<L$ and by using (3.5) and mathematical induction, we can show that $x_{n}^{*} \leq L$ for all $n \geq 1$. Thus, we have $\left|x_{n}^{*}-p\right| \leq\left|x_{n}-p\right|$ for all $n \geq 1$. It follows that $\lim _{n \rightarrow \infty} x_{n}^{*}=p$ and $\left\{x_{n}^{*}\right\}_{n=1}^{\infty}$ converges faster than $\left\{x_{n}\right\}_{n=1}^{\infty}$.

Case 3: $L \leq x_{1}^{*}=x_{1} \leq U$. Suppose that $T x_{1}^{*} \neq x_{1}^{*}$. Without loss of generality, we suppose $T x_{1}^{*}<x_{1}^{*}$. It follows by (3.5) that $x_{n+1} \leq x_{n}$ for all $n \geq 1$. Since $\lim _{n \rightarrow \infty} x_{n}=p$, we must get $p<x_{1}^{*}=x_{1}$. By the same argument as in Case 1 , we have $p \leq x_{n}^{*} \leq x_{n}$ for all $n \geq 1$. It follows that $\left|x_{n}^{*}-p\right| \leq\left|x_{n}-p\right|$ for all $n \geq 1$. Hence, $\lim _{n \rightarrow \infty} x_{n}^{*}=p$ and $\left\{x_{n}^{*}\right\}_{n=1}^{\infty}$ converges faster than $\left\{x_{n}\right\}_{n=1}^{\infty}$.

Finally, we present two numerical examples for comparing rate of convergence between $W^{(T, N)}$-iteration and $K^{(T, N)}$-iteration. 
Table 1 Comparison of the rate of convergence between $W^{(T, 10)}$-iteration and the $K^{(T, 10)}$ iteration for the mapping given in Example 3.6, for $\lambda_{n, i}=\frac{1}{n^{1.5}+1}(i=1,2, \ldots, 9)$ and $\lambda_{n, 10}=\frac{1}{n^{0.5}+1}$

\begin{tabular}{ccccccc}
\hline & \multicolumn{3}{c}{$W^{(T, 10)}$-iteration } & \multicolumn{3}{c}{$\boldsymbol{K}^{(T, 10)}$-iteration } \\
\cline { 2 - 7 }$n$ & $u_{n}$ & $\left|T u_{n}-u_{n}\right|$ & $\left|\frac{u_{n}-u_{n-1}}{u_{n}}\right|$ & $\boldsymbol{x}_{\boldsymbol{n}}$ & $\left|T \boldsymbol{x}_{\boldsymbol{n}}-\boldsymbol{x}_{\boldsymbol{n}}\right|$ & $\left|\frac{\boldsymbol{x}_{n}-x_{n-1}}{x_{n}}\right|$ \\
\hline 5 & 3.148579041 & $4.2578 \mathrm{E}-01$ & $5.1938 \mathrm{E}-02$ & 4.038406568 & $3.8114 \mathrm{E}-03$ & $1.3069 \mathrm{E}-03$ \\
$\vdots$ & $\vdots$ & $\vdots$ & $\vdots$ & $\vdots$ & $\vdots$ & $\vdots$ \\
41 & 4.007635152 & $1.7228 \mathrm{E}-02$ & $6.1858 \mathrm{E}-04$ & 4.047140965 & $2.4503 \mathrm{E}-05$ & $1.0900 \mathrm{E}-06$ \\
42 & 4.009942844 & $1.6218 \mathrm{E}-02$ & $5.7549 \mathrm{E}-04$ & 4.047144996 & $2.2757 \mathrm{E}-05$ & $9.9604 \mathrm{E}-07$ \\
43 & 4.012093026 & $1.5277 \mathrm{E}-02$ & $5.3593 \mathrm{E}-04$ & 4.047148686 & $2.1159 \mathrm{E}-05$ & $9.1160 \mathrm{E}-07$ \\
44 & 4.014098247 & $1.4400 \mathrm{E}-02$ & $4.9954 \mathrm{E}-04$ & 4.047152068 & $1.9695 \mathrm{E}-05$ & $8.3559 \mathrm{E}-07$ \\
45 & 4.015969896 & $1.3582 \mathrm{E}-02$ & $4.6605 \mathrm{E}-04$ & 4.047155172 & $1.8351 \mathrm{E}-05$ & $7.6704 \mathrm{E}-07$ \\
\hline
\end{tabular}

Example 3.6 Let $T:[0,8] \rightarrow[0,8]$ be defined by $T x=-\sin \left(\frac{x-3}{2}\right)+x+\frac{1}{2}$. Then $T$ is a continuous and nondecreasing mapping. The comparison of the rate of convergence of the $W^{(T, N)}$-iteration $\left\{u_{n}\right\}_{n=1}^{\infty}$ and $K^{(T, N)}$-iteration $\left\{x_{n}\right\}_{n=1}^{\infty}$ to a fixed point of $T$ are given in Table 1, with the initial point $u_{1}=x_{1}=1$ when $N=10$.

From Table 1, we see that the $K^{(T, 10)}$-iteration converges faster than the $W^{(T, 10)}$-iteration under the same control conditions. We also observe that $x_{45}=4.047155172$ is an approximation of the fixed point of $T$ with accuracy at 6 significant digits.

Example 3.7 Let $T:[-7,7] \rightarrow[-7,7]$ be defined by

$$
T x= \begin{cases}0.7 x+e^{-0.8}+0.8, & \text { if } x \in[-7,-4) \\ e^{\frac{x}{5}}-2, & \text { if } x \in[-4,5) \\ (x-5)^{2}+e-2, & \text { if } x \in[5,7] .\end{cases}
$$

Then $T$ is a continuous and nondecreasing mapping. The comparison of the rate of convergence of the $W^{(T, N)}$-iteration $\left\{u_{n}\right\}_{n=1}^{\infty}$ and $K^{(T, N)}$-iteration $\left\{x_{n}\right\}_{n=1}^{\infty}$ to a fixed point of $T$ are given in Table 2, when $N=12$.

In Example 3.7, the mapping $T$ is continuous on $[-7,7]$ but it not differentiable at $x=$ -4 and $x=5$. In Table 2, we observe that the $K^{(T, 12)}$-iteration and $W^{(T, 12)}$-iteration with the initial point is $x=5$ converge to a fixed point $p \approx-1.215863862$ of $T$. Moreover, the $K^{(T, 12)}$-iteration converges faster than the $W^{(T, 12)}$-iteration.

Table 2 Comparison of the rate of convergence between the $W^{(T, 12)}$-iteration and $K^{(T, 12)}$ iteration for the mapping given in Example 3.7, for $\lambda_{n, i}=\frac{1}{n^{2}+1}(i=1,2, \ldots, 11)$ and $\lambda_{n, 12}=\frac{1}{n^{0.5}+1}$, with the initial point $\boldsymbol{u}_{\mathbf{1}}=\boldsymbol{x}_{\mathbf{1}}=\mathbf{5}$

\begin{tabular}{ccccccc}
\hline & \multicolumn{3}{c}{$\boldsymbol{W}^{(\boldsymbol{T}, 12)}$-iteration } & \multicolumn{3}{c}{$\boldsymbol{K}^{(\boldsymbol{T}, 12)}$-iteration } \\
\cline { 2 - 7 } $\boldsymbol{n}$ & $\boldsymbol{u}_{\boldsymbol{n}}$ & $\left|T u_{n}-u_{n}\right|$ & $\left|\frac{u_{n}-u_{n-1}}{u_{n}}\right|$ & $\boldsymbol{x}_{\boldsymbol{n}}$ & $\left|T \boldsymbol{x}_{\boldsymbol{n}}-\boldsymbol{x}_{\boldsymbol{n}}\right|$ & $\left|\frac{x_{n}-\boldsymbol{x}_{n-1}}{x_{n}}\right|$ \\
\hline 5 & -0.343578991 & $7.2283 \mathrm{E}-01$ & $8.7516 \mathrm{E}-01$ & -1.215808886 & $4.6467 \mathrm{E}-05$ & $4.2767 \mathrm{E}-05$ \\
$\vdots$ & $\vdots$ & $\vdots$ & $\vdots$ & $\vdots$ & $\vdots$ & $\vdots$ \\
27 & -1.202983546 & $1.0858 \mathrm{E}-02$ & $1.6864 \mathrm{E}-03$ & -1.215863785 & $1.7805 \mathrm{E}-07$ & $2.9928 \mathrm{E}-08$ \\
28 & -1.204709686 & $9.4031 \mathrm{E}-03$ & $1.4328 \mathrm{E}-03$ & -1.215863815 & $1.5237 \mathrm{E}-07$ & $2.5043 \mathrm{E}-08$ \\
29 & -1.206182603 & $8.1616 \mathrm{E}-03$ & $1.2211 \mathrm{E}-03$ & -1.215863841 & $1.3080 \mathrm{E}-07$ & $2.1041 \mathrm{E}-08$ \\
30 & -1.2074442871 & $7.0994 \mathrm{E}-03$ & $1.0437 \mathrm{E}-03$ & -1.215863862 & $1.1261 \mathrm{E}-07$ & $1.7746 \mathrm{E}-08$ \\
\hline
\end{tabular}


Open Problem: Is it possible to prove the convergence theorem of a finite family of continuous mappings on an arbitrary interval by using $W$-mappings and $K$-mappings and how about the rate of convergence of those methods?

\section{Acknowledgements}

The authors would like to thank the Centre of Excellence in Mathematics, the Commission on Higher Education for financial support. WP is supported by the Office of the Higher Education Commission and the Graduate School of Chiang Mai University, Thailand.

\section{Author details}

${ }^{1}$ Department of Mathematics, Faculty of Science, Chiang Mai University, Chiang Mai 50200, Thailand ${ }^{2}$ Centre of Excellence in Mathematics, CHE, Si Ayutthaya Road, Bangkok 10400, Thailand

\section{Authors' contributions}

All authors contributed equally and significantly in this research work. All authors read and approved the final manuscript.

\section{Competing interests}

The authors declare that they have no competing interests.

\section{Received: 6 October 2011 Accepted: 31 January 2012 Published: 31 January 2012}

\section{References}

1. Mann, WR: Mean value methods in iteration. Proc Amer Math Soc. 4, 506-510 (1953). doi:10.1090/S0002-9939-19530054846-3

2. Ishikawa, S: Fixed points by a new iteration method. Proc Amer Math Soc. 44, 147-150 (1974). doi:10.1090/S0002-99391974-0336469-5

3. Noor, MA: New approximation schemes for general variational inequalities. J Math Anal Appl. 251, 217-229 (2000). doi:10.1006/jmaa.2000.7042

4. Phuengrattana, W, Suantai, S: On the rate of convergence of Mann, Ishikawa, Noor and SP-iterations for continuous functions on an arbitrary interval. J Comput Appl Math. 235, 3006-3014 (2011). doi:10.1016/j.cam.2010.12.022

5. Rhoades, BE: Comments on two fixed point iteration methods. J Math Anal Appl. 56, 741-750 (1976). doi:10.1016/0022247X(76)90038-X

6. Borwein, D, Borwein, J: Fixed point iterations for real functions. J Math Anal Appl. 157, 112-126 (1991). doi:10.1016/ 0022-247X(91)90139-Q

7. Qing, Y, Qihou, L: The necessary and sufficient condition for the convergence of Ishikawa iteration on an arbitrary interval. J Math Anal Appl. 323, 1383-1386 (2006). doi:10.1016/j.jmaa.2005.11.058

8. Atsushiba, S, Takahashi, W: Strong convergence theorems for a finite family of nonex-pansive mappings and applications, in: B.N. Prasad Birth Centenary Commemoration Volume. Indian J Math. 41, 435-453 (1999)

9. Ceng, LC, Cubiotti, P, Yao, JC: Strong convergence theorems for finitely many nonexpansive mappings and applications Nonlinear Anal. 67, 1464-1473 (2007). doi:10.1016/j.na.2006.06.055

10. Cho, YJ, Kang, SM, Qin, X: Approximation of common fixed points of an infinite family of nonexpansive mappings in Banach spaces. Comput Math Appl. 56, 2058-2064 (2008). doi:10.1016/j.camwa.2008.03.035

11. Cholamjiak, P, Suantai, S: A new hybrid algorithm for variational inclusions, generalized equilibrium problems and a finite family of quasi-nonexpansive mappings. Fixed Point Theory and Applications 2009, 20 (2009). Article ID 350979

12. Colao, V, Marino, G, Xu, H-K: An iterative method for finding common solutions of equilibrium and fixed point problems. J Math Anal Appl. 344, 340-352 (2008). doi:10.1016/j.jmaa.2008.02.041

13. Kangtunyakarn, A, Suantai, S: Hybrid iterative scheme for generalized equilibrium problems and fixed point problems of finite family of nonexpansive mappings. Nonlinear Anal Hybrid Syst. 3, 296-309 (2009). doi:10.1016/j.nahs.2009.01.012

14. Kimura, Y, Takahashi, W: Weak convergence to common fixed points of countable nonexpansive mappings and its applications. J Korean Math Soc. 38, 1275-1284 (2001)

15. Nakajo, K, Shimoji, K, Takahashi, W: Strong convergence to common fixed points of families of nonexpansive mappings in Banach spaces. J Nonlinear Convex Anal. 8, 11-34 (2007)

16. Nakajo, K, Shimoji, K, Takahashi, W: On strong convergence by the hybrid method for families of mappings in Hilbert spaces. Nonlinear Anal. 71, 112-119 (2009). doi:10.1016/j.na.2008.10.034

17. Qin, X, Cho, YJ, Kang, SM: Viscosity approximation methods for generalized equilibrium problems and fixed point problems with applications. Nonlinear Anal. 72, 99-112 (2010). doi:10.1016/j.na.2009.06.042

18. Shimoji, K, Takahashi, W: Strong convergence to common fixed points of infinite nonexpansive mappings and applications. Taiwanese J Math. 5, 387-404 (2001)

19. Singthong, U, Suantai, S: A new general iterative method for a finite family of nonexpansive mappings in Hilbert spaces. Fixed Point Theory and Appl 2010, 12 (2010). Article ID 262691

20. Takahashi, W: Weak and strong convergence theorems for families of nonexpansive mappings and their applications. Ann Univ Mariae Curie-Sklodowska. 51, 277-292 (1997)

21. Takahashi, W, Shimoji, K: Convergence theorems for nonexpansive mappings and feasibility problems. Math Comput Model. 32, 1463-1471 (2000). doi:10.1016/S0895-7177(00)00218-1

22. Yao, Y: A general iterative method for a finite family of nonexpansive mappings. Nonlinear Anal. 66, 2676-2687 (2007). doi:10.1016/j.na.2006.03.047

23. Yao, Y, Noor, MA, Liou, Y-C: On iterative methods for equilibrium problems. Nonlinear Anal. 70, 497-509 (2009). doi:10.1016/..na.2007.12.021 
24. Kangtunyakarn, A, Suantai, S: A new mapping for finding common solutions of equilibrium problems and fixed point problems of finite family of nonexpansive mappings. Nonlinear Anal. 71, 4448-4460 (2009). doi:10.1016/j.na.2009.03.003

25. Onjai-uea, N, Jaiboon, C, Kumam, P: A strong convergence theorem based on a relaxed extragradient method for generalized equilibrium problems. Appl Math Sci. 4, 691-708 (2010)

26. Peathanom, S, Phuengrattana, W: A hybrid method for generalized equilibrium, variational inequality and fixed point problems of finite family of nonexpansive mappings. Thai J Math. 9, 95-119 (2011)

27. Berinde, V: Picard iteration converges faster than Mann iteration for a class of quasi-contractive operators. Fixed Point Theory Appl. 2, 97-105 (2004)

28. Popescu, O: Picard iteration converges faster than Mann iteration for a class of quasi-contractive operators. Math Commun. 12, 195-202 (2007)

29. Soltuz, SM: The equivalence of Picard, Mann and Ishikawa iterations dealing with quasi-contractive operators. Math Commun. 10, 81-88 (2005)

30. Babu, GV, Prasad, KN: Mann iteration converges faster than Ishikawa iteration for the class of Zamfirescu operators. Fixed Point Theory and Appl 2006, 6 (2006). Article ID49615

31. Qing, Y, Rhoades, BE: Comments on the Rate of Convergence between Mann and Ishikawa Iterations Applied to Zamfirescu Operators. Fixed Point Theory and Appl 2008, 3 (2008). Article ID 387504

doi:10.1186/1687-1812-2012-9

Cite this article as: Phuengrattana and Suantai: Strong convergence theorems and rate of convergence of multistep iterative methods for continuous mappings on an arbitrary interval. Fixed Point Theory and Applications 2012 2012:9.

\section{Submit your manuscript to a SpringerOpen ${ }^{\circ}$} journal and benefit from:

- Convenient online submission

Rigorous peer review

- Immediate publication on acceptance

- Open access: articles freely available online

- High visibility within the field

- Retaining the copyright to your article

Submit your next manuscript at $\boldsymbol{\triangleright}$ springeropen.com 